\title{
Discharge Patterns of the Primary Auditory Cortex in Cats
}

\author{
Masahiro Noмото \\ Department of Physiology, Medical School, Dokkyo \\ University, Tochigi, 321-02 Japan
}

\begin{abstract}
Single unit responses of the primary auditory cortex of unanesthetized cats were studied using three standard sets of sound stimuli, i.e., clicks, white noise bursts, and pure tone bursts. The units studied were classified into eight categories according to whether they responded to all three, two, one or none of the standard sets of stimuli; there were no units responding only to clicks. This suggests that excitation of some cortical neurons requires a special sound spectrum as well as temporal factors. Frequency-modulated (FM) sounds were usually more effective eliciting responses than the standard sets of stimuli. Responses to FM in bursts seemed to be classified into three groups, i.e., sharp monopeak post-stimulus time (PST) histograms, broad monopeak, and sharp multipeaks. The existence of the last group suggests a firm convergence from the lower auditory neurons. In some units, complex stimuli were more effective in evoking responses than simple FM sounds. These units responded only to sounds with complex temporal patterns. For example, a neuron did not respond to any FM sound with a constant modulation magnitude, but showed a transient response whenever the magnitude of FM varied from a continuous pure tone to a certain range of FM. These complex units often showed a response with hysteresis.
\end{abstract}

Neurons of the primary auditory cortex (AI) in mammals show remarkable specificities in their response patterns to tonal stimulation. What mainly distinguishes the cortical neurons from others at the lower level of the auditory nervous system is the great complexity of their responsiveness in which the temporal aspect of sound stimulation is particularly important because of the ineffectiveness of monotonic stimuli. However, what parameters are effective is not yet clear at the higher level of the auditory system, especially at the auditory cortex, since the small size of the neuron somata in the cortex and subtle sound parameters make experimentation difficult. There have been many investigations (ABELES and Goldstein, 1970; Goldstein et al., 1970; EvAns and Whitfield, 1964; FunKenstein and WinTER, 1973; Goldstein et al., 1968; GoldDSTEIN and ABELES,

Received for publication September 14, 1979

野本昌弘 
1975; KATSUKi et al., 1959, 1962; Oonishi and KATSUKI, 1965; SoviJARVI and Hyvarinen, 1974; Suga, 1965a, b; Whitfield and Evans, 1965; Winter and FUNKENSTEIN, 1973; Wollberg and NewMAN, 1972), but the role of individual neurons in the processing of auditory information is little understood.

The use of frequency-modulated tone (FM) as a stimulus would be equivalent in some respects to a moving slit of light in the visual system (HUBEL and WIESEL, 1965) in terms of spatial cell arrangements in both systems, because sound frequency could be considered to be spatially assigned to neurons along the basilar membrane. The purpose of the present study was to describe the acoustic parameters effective in the neuron of the primary auditory cortex of the cat.

\section{METHODS}

Cats, weighing 2-4 kg, unanesthetized, were used to search for single unit responses in the primary auditory cortex. The bone above area AI was removed and a stainless steel cylinder was implanted in a $10-15 \mathrm{~mm}$ hole through the skull, using dental acrylic with the aid of three machine screws. The wound was closed with surgical sutures. Local anesthetics were applied to the wound. Semichronic experiments were performed with the mount of the microelectrode holder fixed over the AI of cats. A tungsten microelectrode was inserted into the cortex by remote control either through the dura mater or after the dura mater had been removed. The animals were initially anesthetized with sodium pentobarbital, given intraperitoneally, in a dose of $25 \mathrm{mg} / \mathrm{kg}$. The insertion of the tungsten microelectrode was begun 12 to $18 \mathrm{hr}$ after injection.

Sound stimuli were delivered into a free field in a shielded sound-proofed room. The sound pressure level was calibrated with a Bruel and Kjaer condenser microphone placed very close to the test ear. Throughout this paper, the relative sound pressure level is $0 \mathrm{~dB}=93.8 \mathrm{~dB}$ SPL (relative to $0.0002 \mathrm{dyn} / \mathrm{cm}^{2}$ ) at $1 \mathrm{kHz}$. As the most fundamental sound stimuli, clicks, white noise bursts and pure tone bursts, were used with a repetition rate of about $1 \mathrm{sec}$. The white noise bursts and pure tone bursts had a duration of approximately $100 \mathrm{msec}$ and a rise and fall time of $5 \mathrm{msec}$. Two kinds of clicks, of $10-\mu \mathrm{sec}$ or $100-\mu \mathrm{sec}$ duration, were generated by a square pulse generator. Frequency-modulated (FM) sounds were also applied as stimuli. Pure tone bursts were modulated linearly in an ascending or descending mode and continuous pure tones were modulated by sine waves. These are usually called FM bursts and continuous sine wave FM, respectively.

\section{RESULTS}

Responses to clicks, white noise bursts, and pure tone bursts

Three standard sets of sound stimuli, i.e., clicks, white noise bursts, and pure tone bursts, were used in order to classify single unit responses of the primary auditory cortex in cats, although the cortical neurons are very sensitive to a 
transient stimulus and not to a steady stimulus. Temporal patterns of responses of the neurons to these pure tones and white noise bursts showed on, off, on-off, on-sustained and off-sustained excitatory responses, and inhibitory ones. Of course, during the inhibitory action spontaneous discharge was suppressed. It became clear in many cases that single neuron responses showed different discharge patterns according to the stimulus.

The units studied were classified categorically as responding to all three, two, one or none of these stimuli applied separately; no unit responded only to clicks that were generated as either $10-\mu \mathrm{sec}$ or $100-\mu \mathrm{sec}$ duration square pulses. This is shown in Table 1 for 107 units. Of eight possible combinations of the three sounds, seven combinations were effective in eliciting responses. Thirty-six percent of the single neurons obtained from the AI responded to all three stimuli. It was possible that the characteristic responsiveness of these neurons could be sensitive to some factors common in the three sets of stimuli. The second largest group (about $20 \%$ ) responded exclusively to pure tone bursts. The third largest group (about 19\%) responded to none of these stimuli. The fourth group (10\%) was responsive to both white noise bursts and pure tone bursts, and so on. This fact suggests that the neurons can respond to a common feature in temporal terms or frequency or both components of these sounds. It is not understood why units responding only to clicks were not found. Pure tone bursts were the most effective of the three sets of stimuli.

\section{Responses to frequency modulation (FM)}

FM sounds were used to elicit responses of the neurons in the AI. Simple FM bursts were not so effective as at the lower level of auditory neurons. It was very difficult to find the acoustic parameters in the AI. However, FM stimuli were generally more effective than pure tone bursts. Many units were relatively more sensitive to the continuous sine wave FM than to the FM bursts irrespective of response patterns to the three sets of stimuli. Continuous FM sounds effec-

Table 1. Effect on cortical neurons of three sets of sound stimuli.

\begin{tabular}{cccc}
\hline & Response & & Number of Units \\
\hline Click & WNB & PTB & \\
\hline+ & + & + & 39 \\
+ & - & + & 5 \\
+ & + & + & 3 \\
- & - & - & 11 \\
+ & + & + & 0 \\
- & - & - & 21 \\
- & - & 20 \\
\hline
\end{tabular}

+ indicates response to stimuli, - indica
white noise bursts. PTB: pure tone bursts.

Vol. 30, No. 3, 1980 

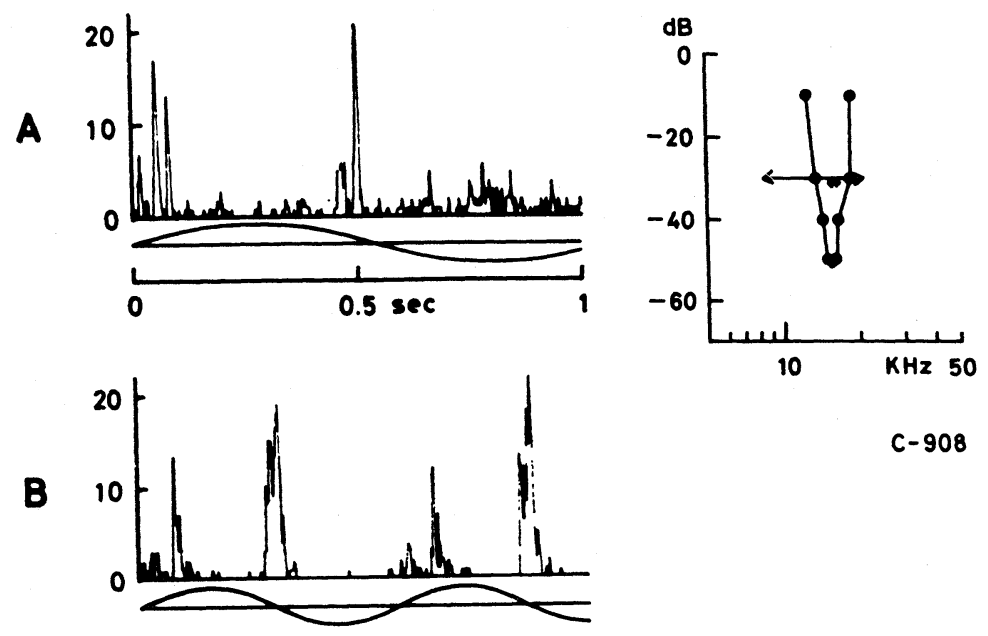

C-908
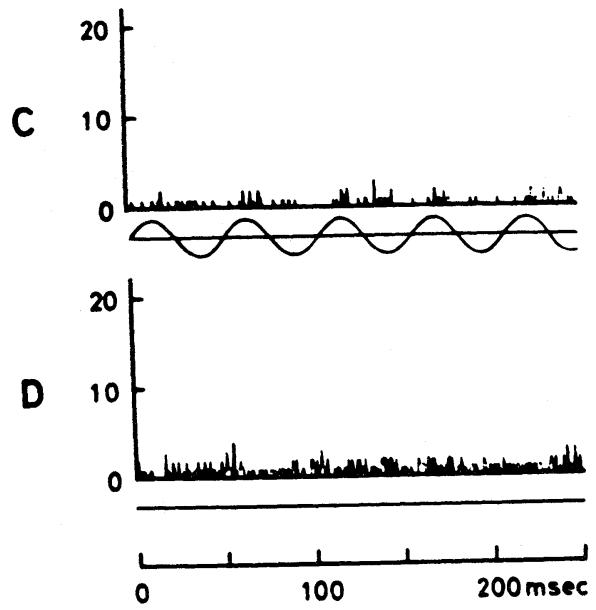

Fig. 1. PST histograms of responses to continuous sine wave frequency modulation from 8.57 to $20.6 \mathrm{kHz}$ at $-30 \mathrm{~dB}$. Abscissae and ordinates represent number of impulses in the period of $125 \mathrm{msec}$ and time, respectively. A: modulation frequency, $0.9 \mathrm{~Hz}$. B: modulation frequency, $7 \mathrm{~Hz}$. C: modulation frequency, $20 \mathrm{~Hz} . \quad$ D: $14.2 \mathrm{kHz}$ continuous pure tone. This frequency modulation is shown as curves under graphs A, B and $\mathrm{C}$.

tive to the neurons were modulated appropriately by sine waves with a 50 -msec to 3 -sec period. FM sounds with an optimum sweeping rate and a certain range of frequencies were usually necessary. Figure 1 shows post-stimulus time (PST) histograms. Unless otherwise specified, total sampling time for one bin is 125 msec for all the following PST histograms, which have four hundred bins. In Fig. $1 \mathrm{~A}$, the number of trials was 50 and the sampling time was $1 \mathrm{sec}$, while in Fig. 1B, C and D the number of trials was 200 and the sampling time was 250 
msec, so that total sampling time of one bin was always maintained at $125 \mathrm{msec}$. In this example, the sweep range was from 8.57 to $20.6 \mathrm{kHz}$ at $-30 \mathrm{~dB}$. In Fig. $1 \mathrm{~A}, \mathrm{~B}$ and $\mathrm{C}$, the modulation frequencies were approximately $0.9,7$ and $20 \mathrm{~Hz}$, respectively. At $20 \mathrm{~Hz}$ there was little excitation of the unit. Figure 1D shows the unit response to a continuous pure tone of $14.2 \mathrm{kHz}$ but the response barely exceeded the level of spontaneous discharge. The unit response occurred when FM sounds either crossed the boundaries of its tuning curve or were confined within the tuning curve as indicated by bars with arrows, where the tuning curve was measured by the use of pure tone bursts. This is shown on the right side of
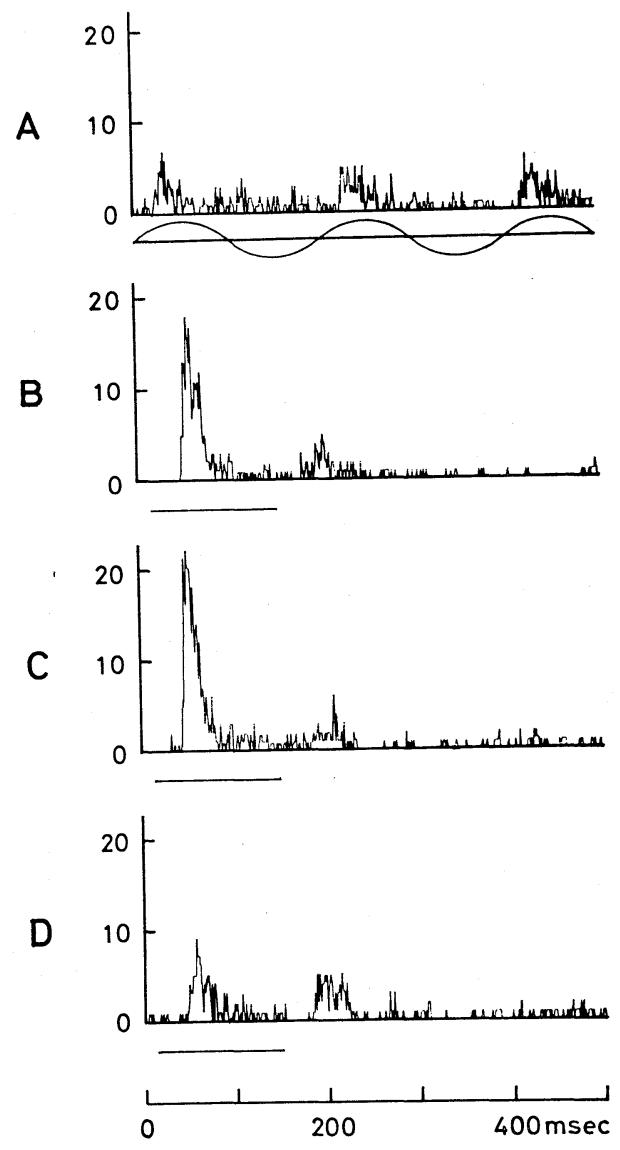

C -603

Fig. 2. Responses to a continuous FM sound and pure tone bursts. A: continuous sine wave modulation with $5 \mathrm{~Hz}$ from 5.7 to $8.9 \mathrm{kHz}$ at $-20 \mathrm{~dB}$. Ordinate: number of impulses in the period of $125 \mathrm{msec}$. B, C and D: responses to pure tone stimuli with $5.7,7.4$ and $8.9 \mathrm{kHz}$ at $-20 \mathrm{~dB}$, respectively.

Vol. 30, No. 3, 1980 
Fig. 1. There is a suitable range and modulation rate of FM to elicit responses, as shown in Fig. 1.

On the contrary, continuous sine wave FM by $5 \mathrm{~Hz}$ elicited a few responses in the unit shown in Fig. 2A, although pure tone bursts evoked a large response. In this unit, white noise bursts and click stimuli were ineffective. The frequency range of sine wave FM was from 5.7 to $8.9 \mathrm{kHz}$ at $-20 \mathrm{~dB}$. This range was well within the response area of this unit. Frequencies of pure tone bursts were 5.7, 7.4 , and $8.9 \mathrm{kHz}$ at $-20 \mathrm{~dB}$ as shown in Fig. $2 \mathrm{~B}, \mathrm{C}$ and $\mathrm{D}$, respectively. The response range of frequency of pure tone bursts was 0.5 to $18 \mathrm{kHz}$ at $-20 \mathrm{~dB}$. In this situation, responses to pure tone bursts were stronger than to continuous sine wave FM. Therefore, modulation frequency and range, i.e., sweeping rate of FM, are important in evoking response. In the AI, directional sensitivity to FM is not so dominant in nature, although the directional sensitivity is relatively clear at the lower level of the auditory system.

However, it was more difficult to elicit a response with FM bursts than with

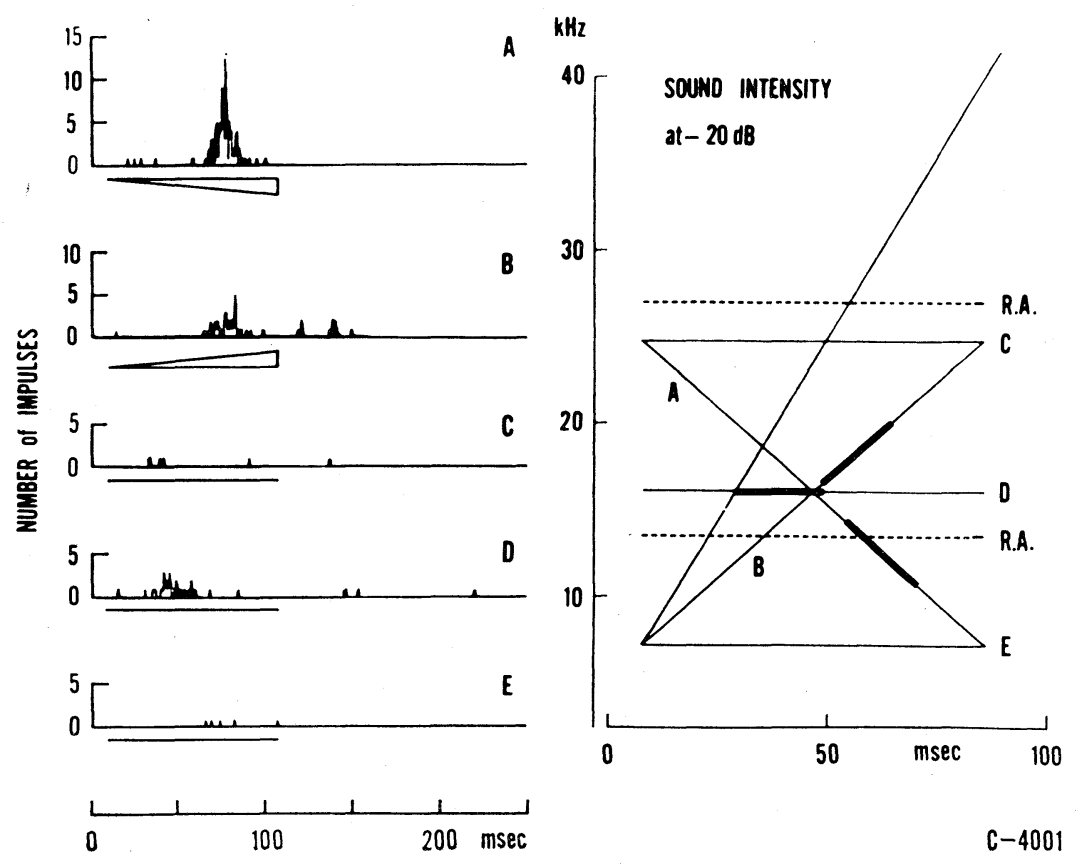

Fig. 3. PST histograms of responses to FM bursts with a relatively sharp monopeak. Ordinates show number of impulses. Number of trials is 100 . Thin lines in the figure at right represent sound stimuli and thick lines show responses. There is different directional sensitivity to FM. A: descending FM bursts from 23 to $7.4 \mathrm{kHz}$ with 100 trials at $-20 \mathrm{~dB}$. B: ascending FM bursts from 7.4 to $23 \mathrm{kHz}$ under the same conditions. C, D and E: pure tone bursts of $23,15.9$ and $7.4 \mathrm{kHz}$, respectively. No response to white noise bursts or clicks. 
continuous FM. Responses to FM bursts were classified into three categories in terms of temporal patterns. First, units sensitive to FM bursts showed a relatively sharp monopeak PST histogram, according to FM bursts with suitable frequency range, as shown in Fig. 3. The area within the two dotted lines on the right side of Fig. 3 shows the limits of the response area at a constant stimulus intensity. On the right side of Fig. 3, the thin lines indicate tone bursts and the thick lines indicate the response to these sounds. In this neuron, a dominant response appeared when the boundary of the response area was crossed in a descending way by an FM burst as shown in Fig. 3A. A suitable frequency modulation rate was required to elicit responses. There seems to be different directional sensitivity to FM tones. A rapid sweeping rate did not cause any response at all. That is, a linear modulation rate of $440 \mathrm{kHz}$ per second was too fast to elicit any response, while $330 \mathrm{kHz}$ per second modulation elicited a response in this particular case.

The second type of unit sensitive to FM bursts showed a monotonously broad monopeak PST histogram without showing any irregular peak at a special frequency, as shown in Fig. 4. FM bursts from 20.3 via 32 to $20.3 \mathrm{kHz}$ caused a sustained response as shown in Fig. 4D, while FM bursts from 19.5 via 7.6 to $19.5 \mathrm{kHz}$ evoked a broad monotonous off-response as seen in Fig. 4F. In this unit, continuous sine wave FM also showed a monotonously broad monopeak tendency, which may correspond to the flat type of OoNISHI and KATSUKI (1965), especially as seen in Fig. 4N.

Thirdly, an interesting unit was found as shown in Fig. 5. This unit represented characteristic multipeak PST histograms. Each peak was clearly dependent upon the frequencies that were crossed by the FM burst. The magnitude in the response of PST histograms may reflect the effect of the lower level neurons which would converge onto the auditory cortical neuron. Various excitatory and inhibitory interferences were present as observed in the increase or decrease of the succeeding peaks depending on the direction and rate of modulation. In Fig. 5, peaks a, b, c, d, e and f were distinguishable due to several FM stimuli. Data collected using the same stimuli during various periods some time apart showed similar patterns of responses. Therefore, there must be some rigid connection between the auditory neurons. On the right side of Fig. 5, relations between the modulated frequency and the time of various stimuli are shown, where thin lines indicate courses of frequency with time of sound stimuli and thick bars on the thin lines represent the presence of responses to these stimuli. Responses were generated when stimuli crossed certain frequency ranges. In these histograms the preceding peaks seem to have strong inhibitory or excitatory effects on the following peaks. This observation may be explained at least qualitatively by the neuronal circuit, for example, one as shown in Fig. 6. Here, neurons $\mathrm{a}, \mathrm{b}, \mathrm{c}, \mathrm{d}$, e and $\mathrm{f}$ at the lower auditory system converge on the neuron studied and interact with each other. Actually, some calculations were carried 


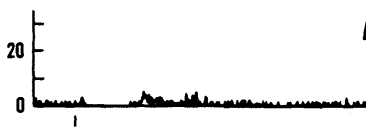

A 5 H

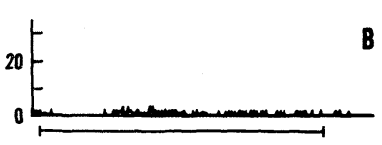

B
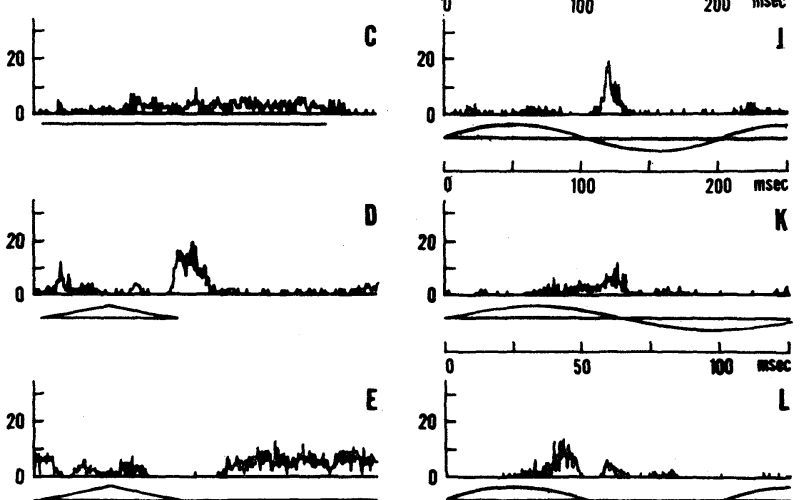

E
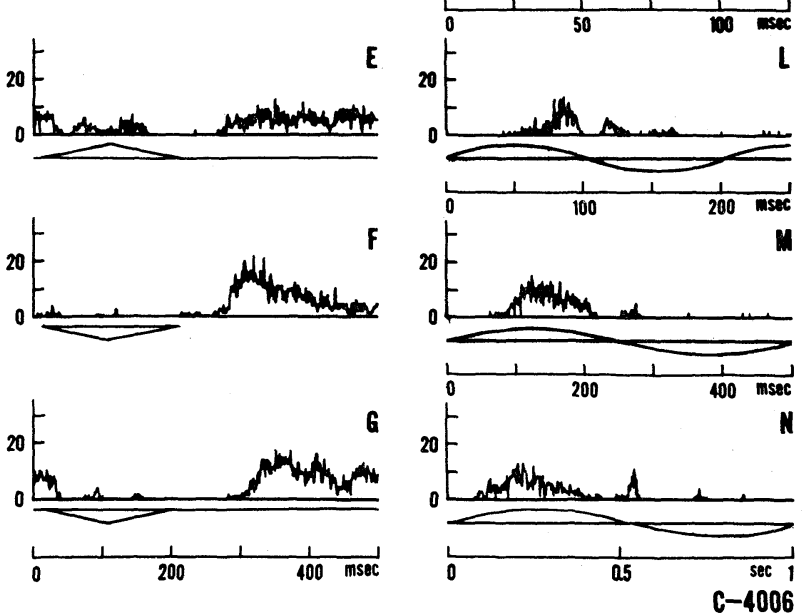

Fig. 4. PST histograms of a broad monopeak unit. A: clicks. B: white noise bursts. C: $20.6 \mathrm{kHz}$ pure tone bursts. D: FM bursts from 20.3 via 32 to $20.3 \mathrm{kHz}$. E: FM bursts as in $\mathrm{D}$ with a continuous pure tone $(20.3 \mathrm{kHz})$. F: FM bursts from 19.5 via 7.6 to $19.5 \mathrm{kHz}$. G: FM bursts as in $\mathrm{F}$ with a continuous pure tone $(19.5 \mathrm{kHz})$. A to $\mathrm{G}$ : sound intensity, $-30 \mathrm{~dB}$; trial number, 100 . $\mathrm{H}$ : spontaneous discharge. I: continuous sine wave FM from 18 to $22.2 \mathrm{kHz}$; modulation frequency, approximately $5 \mathrm{~Hz}$. J: the same as I except frequency range (from 13.6 to $27 \mathrm{kHz}$ ). $\mathrm{K}$ : continuous sine wave FM from 8.5 to $33.5 \mathrm{kHz}$; modulation frequency, approximately $8 \mathrm{~Hz}$. L: continuous sine wave from 8.5 to $33.5 \mathrm{kHz}$; modulation frequency, approximately $5 \mathrm{~Hz}$. M: continuous sine wave FM from 8.5 to $33.5 \mathrm{kHz}$; modulation frequency, approximately $2 \mathrm{~Hz}$. $\mathrm{N}$ : continuous sine wave FM from 8.5 to $33.5 \mathrm{kHz}$; modulation frequency, approximately $1 \mathrm{~Hz}$. Total period of one bin is $125 \mathrm{msec}$ in all PST histograms. 

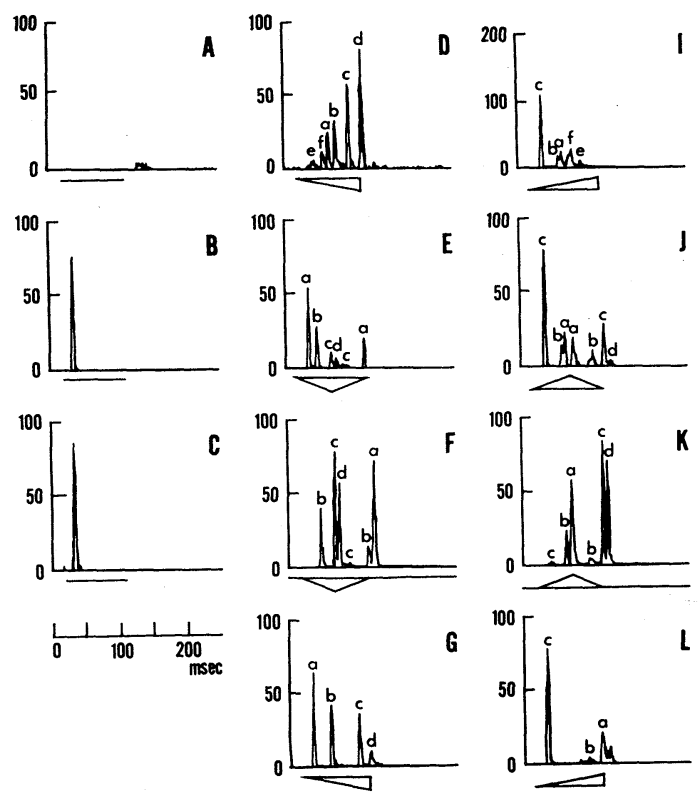

G

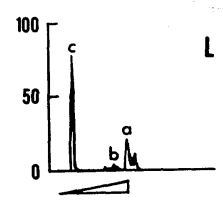

${ }^{\mathrm{kHz}}$

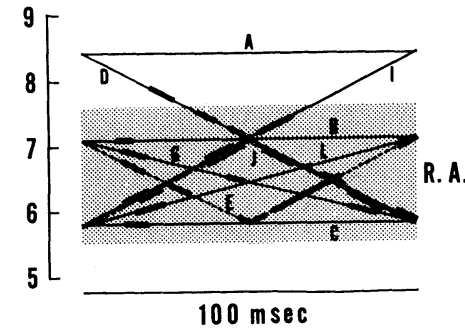

C-4302
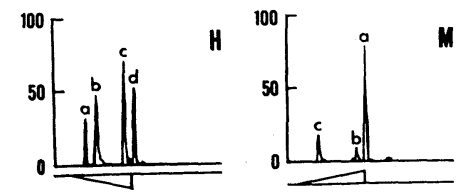

$C-4302$

Fig. 5. PST histograms of a multipeak unit. Ordinate: number of impulses. A, B and $\mathrm{C}$ : pure tone burst stimuli; $8.4,7.1$ and $5.8 \mathrm{kHz}$, respectively. $\mathrm{D}$ : FM bursts from 8.4 to $5.8 \mathrm{kHz}$. E: triangle FM bursts from 7.1 via 5.8 to $7.1 \mathrm{kHz}$; duration, $100 \mathrm{msec}$. $\mathrm{F}$ : triangle FM bursts from 7.1 via 5.8 to $7.1 \mathrm{kHz}$ with a continuous pure tone $(7.1 \mathrm{kHz})$. G: FM bursts from 7.1 to $5.8 \mathrm{kHz}$ with a continuous pure tone $(7.1 \mathrm{kHz})$. I: $\mathrm{FM}$ bursts from 5.8 to $8.4 \mathrm{kHz}$. K: triangle FM bursts from 5.8 via 7.1 to $5.8 \mathrm{kHz}$ with a continuous pure tone $(5.8 \mathrm{kHz})$. L: triangle FM bursts from 5.8 via 7.1 to $5.8 \mathrm{kHz}$. M: FM bursts from 5.8 to $7.1 \mathrm{kHz}$ with a continuous pure tone $(5.8 \mathrm{kHz})$. Sound intensity, $-30 \mathrm{~dB}$. Response band width, 5.5 to $7.6 \mathrm{kHz}$ at $-30 \mathrm{~dB}$. There are responses to white noise bursts. No response to clicks.

out based on the magnitude of each peak, to express the magnitude of the effect by the sizes of the synapses, in this schema. Since the magnitude of each peak was influenced in an excitatory and inhibitory way by the preceding peaks, simultaneous equations which described the magnitudes of each peak as several members of the equations, were constructed to elucidate the relationship between the magnitudes of each peak. Each equation cosisted of addition of its several members that corresponded to the magnitudes of each peak in a given type of stimuli. Solving the simultaneous equations gave the members certain numerals whose positive values indicated an excitatory effect and negative values indicated an 


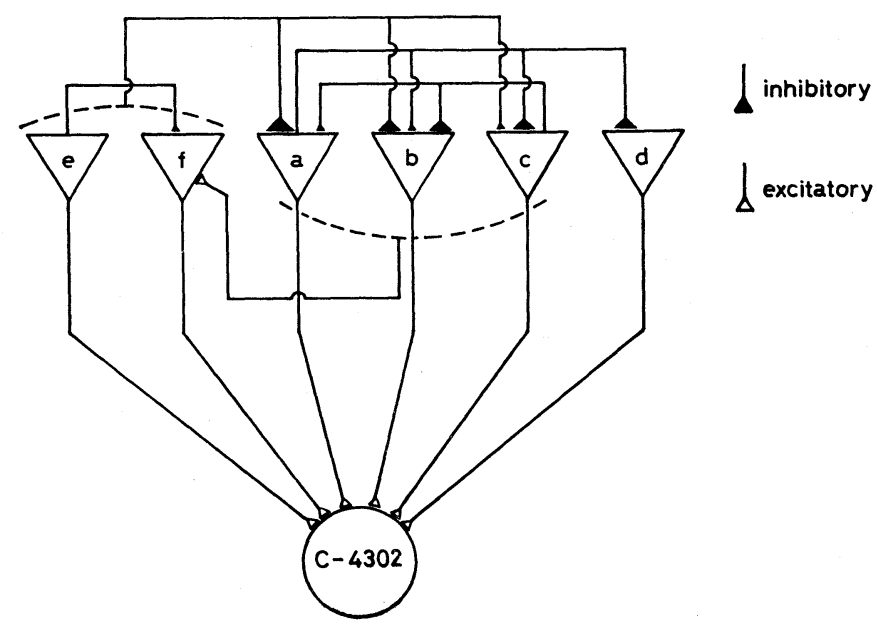

Fig. 6. Schema derived from the neuron of Fig. 5. The auditory neurons of the lower level converge on the cortical neuron C-4302. A large synapse indicates a large effect.

inhibitory effect. In this hypothesis, the possibility of presynaptic inhibition was not considered. These cortical units were obtained in the deep layer of the cortex. These distinctive phenomena were observed in several units. This type of neuron is presumed to correspond to the unit with a multipeak response area (Abeles and Goldstein, 1970; OONISHI and Katsuki, 1965). This type of neuron was also observed in medial geniculate neurons (unpublished observation).

As can be seen in Fig. 7, an FM burst which was preceded by a pure tone of several hundred msec activates responses, presumably because the pure tone removed an on-inhibitory effect of the FM burst. I believe that a large amount of on-inhibitory effect obscures the response to FM bursts. The pure tone burst itself also has an on-inhibitory effect, and this effect continues for several hundred msec so that a prefixed pure tone burst of less than $240 \mathrm{msec}$ did not evoke strong response to FM bursts because of the long-lasting on-inhibitory effect of the pure tone. Figure 7A shows a very weak response but Fig. 7B, E and F show strong responses because of the prefixed pure tone.

On the other hand, an on-excitatory effect that affected responses to an FM burst was also observed. The preceding pure tone inhibited the response to FM stimuli as seen in Fig. 8C. This unit did not respond to any pure tone bursts and clicks, but responded to white noise bursts. These on-excitatory units were observed less frequently than on-inhibitory ones. All the units mentioned above could be classified as simple units, and were in the majority.

\section{Complex units}

These units did not respond to any FM stimuli or to the other three standard sets of sound stimuli. They were defined conventionally as complex units which 

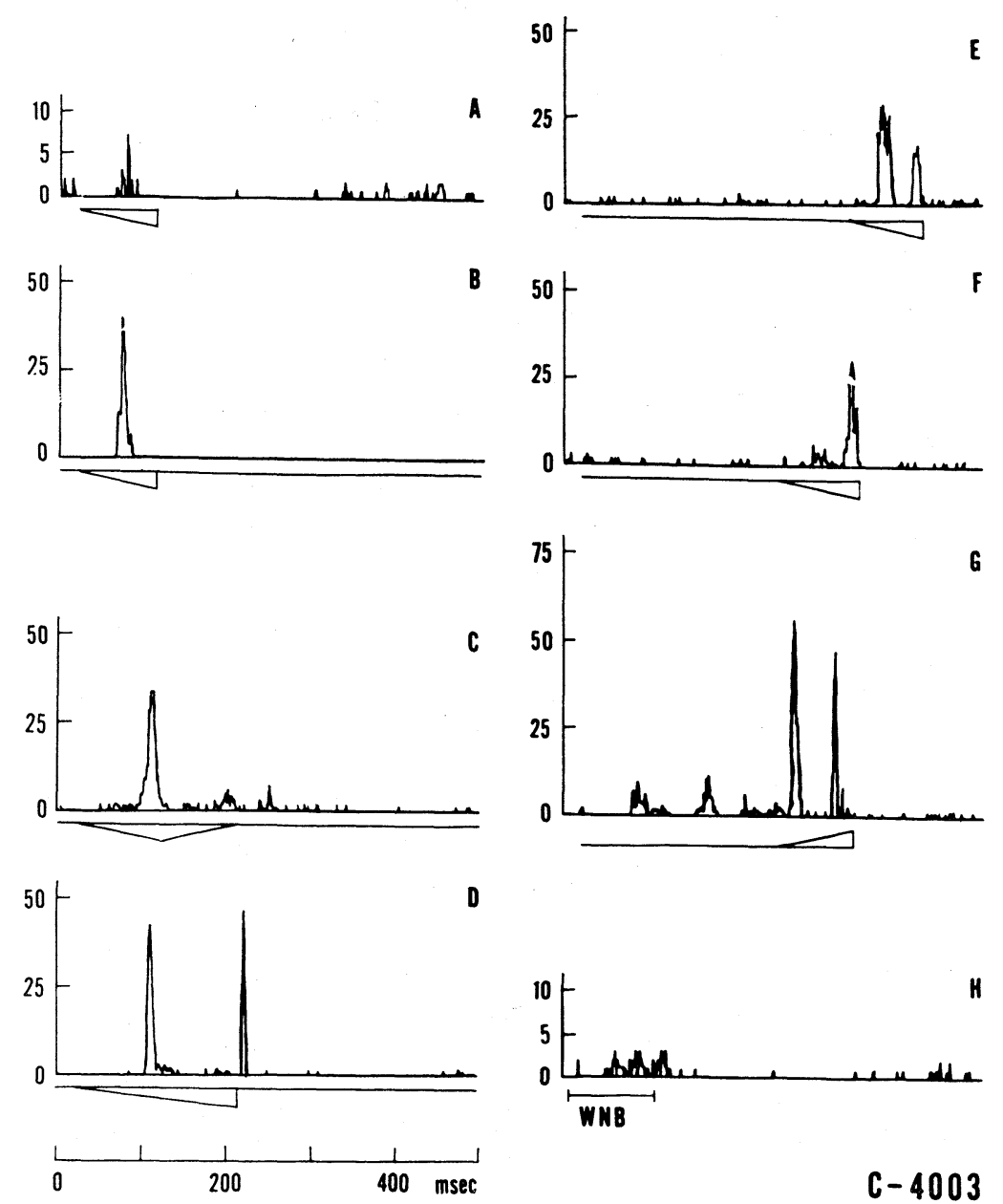

Fig. 7. Effect of pure tones prefixed to FM bursts. A: FM bursts from 13.5 to $3.16 \mathrm{kHz}$. B: FM bursts from 13.5 to $3.16 \mathrm{kHz}$ with a continuous pure tone $(13.5 \mathrm{kHz})$. C: triangle FM bursts with a continuous pure tone $(13.5 \mathrm{kHz})$. D: sawtooth FM bursts with a continuous pure tone $(13.5 \mathrm{kHz})$. E: FM burst from 13.5 to $3.16 \mathrm{kHz}$ with a prefixed pure tone $(13.5 \mathrm{kHz})$ of $330 \mathrm{msec}$ duration. F: the same as $E$ except for the $240 \mathrm{msec}$ duration of the prefixed pure tone. G: FM bursts from 3.15 to $13.5 \mathrm{kHz}$ with a prefixed pure tone $(3.16 \mathrm{kHz})$ of $240 \mathrm{msec}$. H: white noise bursts. Sound intensity A to $\mathrm{G}$ : $-10 \mathrm{~dB}$ and $\mathrm{H}$ : $-20 \mathrm{~dB}$.

could respond only to sound stimuli with a complex temporal pattern. Some were recorded from the posterior part of the primary auditory cortex. It was not easy to confirm factors in the stimulus which were responsible for producing a response in the complex units. The two units shown in Fig. 9 did not respond to any sound stimuli except a transient frequency change in a continuous tone. Unit C-1002 responded transiently only when a continuous tone became an FM sound. 

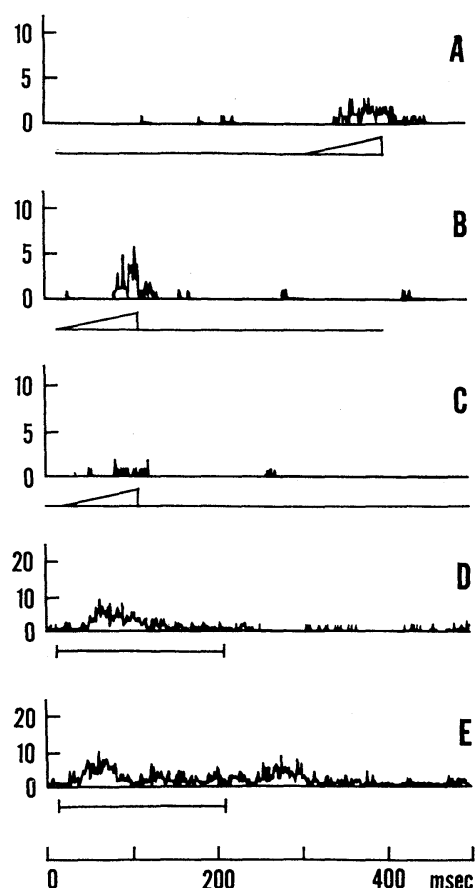

PTB (-)

CLICK (-)

\section{C-4008}

Fig. 8. Effect of on-excitatory action after prefixing a pure tone. A: after prefixing a pure tone of $1.9 \mathrm{kHz}$, response to $\mathrm{FM}$ bursts from 1.9 to $4.7 \mathrm{kHz}$ at $-10 \mathrm{~dB}$. B: FM bursts from 1.9 to $4.7 \mathrm{kHz}$, before $1.9 \mathrm{kHz}$ pure tone bursts at $-10 \mathrm{~dB}$. C: $\mathrm{FM}$ bursts from 1.9 to $4.7 \mathrm{kHz}$ with a continuous tone of $1.9 \mathrm{kHz}$ at $-10 \mathrm{~dB}$. D and $\mathrm{E}$ : white noise bursts at $-30 \mathrm{~dB}$ and $-50 \mathrm{~dB}$, respectively.
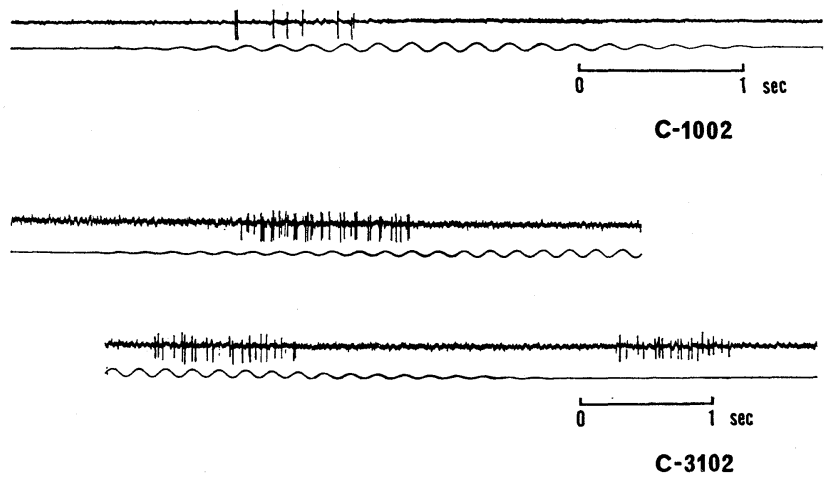

Fig. 9. Responses to transient FM tones. In each set of two lines the upper trace represents response and the lower trace represents the degree of frequency modulation of sound stimuli. 


\section{A}

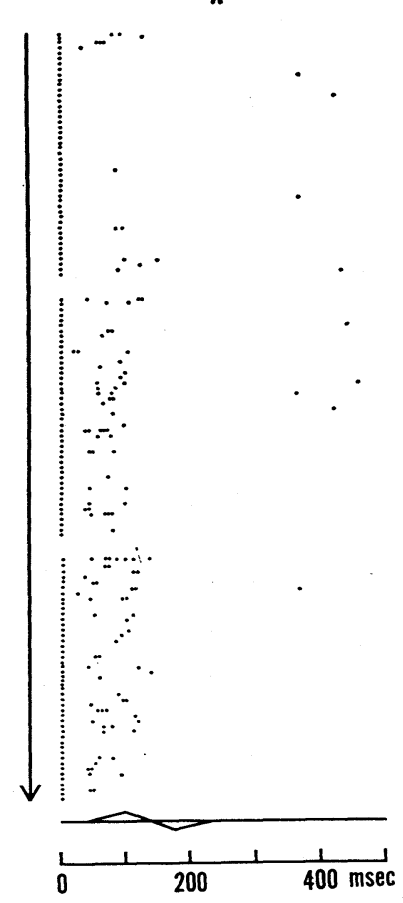

B

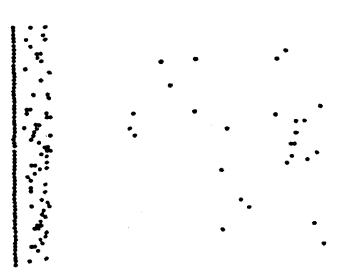

C

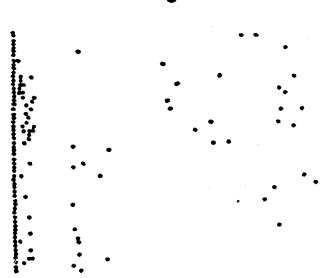

Fig. 10. Dot displays showing nerve impulses. FM bursts are from 1.16 via 1.56 via 0.72 to $1.16 \mathrm{kHz}$. At A, the repetition rate of FM bursts is $2 \mathrm{~Hz}$. At $\mathrm{B}$ and $\mathrm{C}$, the repetition rate is $1 \mathrm{~Hz}$ and $0.5 \mathrm{~Hz}$, respectively.

Unit C-3102 responded only in the case of a continuous tone modified transiently to frequency modulation and vice versa. Steady FM bursts or continuous FM sounds were ineffective in these units.

The neuron shown in the dot display in Fig. 10 may be designated as an expectation unit; the unit responded according to the forecast that the next stimulus will be given. Dots represent nerve impulses. When the FM bursts were applied with regular intervals for a period of time, in the beginning there seemed to be no response at all, but gradually responses appeared preceding the next sound stimuli. A change of the intervals among the repetitive stimuli also caused the same interval change of the responses. Sometimes the regular intervals of the responses became obscure as seen in the last part of Fig. 10, probably because the animal's condition changed. The behavior of this expectation unit is difficult to explain; only one such unit was found.

The unit shown in Fig. 11, did not respond to any pure tone stimuli, but only to white noises and clicks. However, the unit gave an on-off response to a pure tone burst when continuous white noise had been added as a background stimulus. This might indicate an increase of basic activity by a background noise. There 


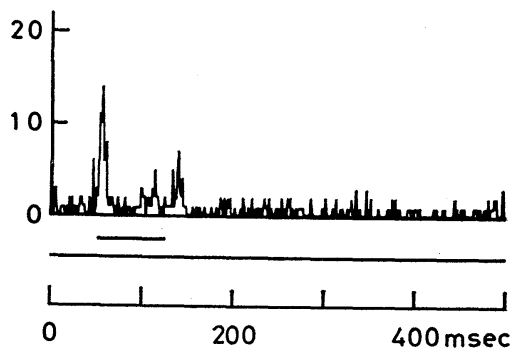

C- 1601

Fig. 11. PST histograms of responses to pure tone bursts of $2.5 \mathrm{kHz}$ at $-20 \mathrm{~dB}$ with a background of continuous white noise at $-30 \mathrm{~dB}$. Ordinates show number of impulses. Under the abscissa, the long line indicates continuous white noise and the short line indicates pure tone bursts.

were three units of this type.

Sometimes it was very difficult to say whether a unit was responding to stimuli or not, because it was often impossible to find proper factors for eliciting a response by an artificial sound.

\section{DISCUSSION}

The three standard sets of acoustic stimuli have features both in common and different. For example, white noise bursts and pure tone bursts have a similar time-course as to their intensity but their frequency spectra differ, so that they evoked responses of a different nature. Temporal discharge patterns to pure tone bursts and white noise bursts differed in the individual neurons. Clicks caused the least discharge, and the temporal aspect of clicks is of course different from that of white noise bursts and pure tone bursts. This means that the spectral components or temporal aspect or both of sounds are important in eliciting responses.

FUNKENSTEIN and WinTER (1973) found that the percentage of units responsive to one or more of the three sets of sound stimuli is approximately $81 \%$ in squirrel monkeys, although small doses of pentobarbital were used in their experiment. My experiments with unanesthetized cats showed the same percentage of $81 \%$. Both experimental conditions were nearly the same. Evans and Whitfield (1964) found responses to pure tone stimuli in $77.5 \%$ of the units obtained from the primary auditory cortex of unanesthetized cats, while GoLDSTEIN et al. (1968) also evoked responses in $95 \%$ of the units in the same preparation. Their higher percentage may be due to the ability to stimulate at higher frequencies than mine and may reflect recording exclusively from AI, while my units were sampled from a broader area. It also seems probable that gallamine, which they used, reduces the proportion of silent cells by its activating effect. 
FM-sensitive units were also observed in the lower auditory system. In this case, directional sensitivities to FM were clearer than those of the AI (SUGA, 1968; WATANABE, 1972). It seems that discrimination of FM directionality is performed in the lower level of the auditory system, so that specific directionality is lost in the auditory cortex. Other factors may be important in the AI. Discharge patterns of auditory cortical neurons did not contain information regarding sound frequency and intensity. This is explained by the shape of response areas and temporal aspects of response discharge. A so-called response area measured with respect to sound intensity and frequency did not seem to have significance in expressing the function of the cortical neurons. In most cases, FM-sensitive units responded to a certain phase of the modulation (WhITFIELD and EvANS, 1965) so that the response occurred when a certain frequency was crossed by the FM tone. This is said on the assumption that responses to various periods of continuous sine wave FM tones have a constant latency.

Particular interest focused on multipeak PST histograms. In such neurons, it is natural to suppose that an integration or convergence of lower auditory neurons, which have sharp tuning curves, occurred on this neuron. This kind of unit seemed to be rather lower in the neural information processing system compared with ordinary cortical neurons, because this type of unit was found dominantly also in the medial geniculate body (unpublished observation). It is also possible that the said cortical neurons might have a direct synapse with such a geniculate neuron.

The units of the auditory cortex often failed to respond to FM bursts. This suggests the existence of inhibition which occurs at the onset of the FM burst stimulus. On the contrary, FM bursts sometimes caused stronger responses than the response to FM bursts with a prefixed pure tone, the pure tone apparently impairing the excitation at the onset of the following FM burst. Such units were far fewer than on-inhibitory units.

Several complex units showed responsiveness to highly sophisticated factors that were mostly temporal. In general, however, it was very difficult to find what were the adequate parameters for eliciting responses in AI. Although sound with wide band frequency components, for example, the animal voice, has been reported to be very effective in evoking responses (WOLlbERG and NeWMAN, 1972; SoviJArvi and Hyvarinen, 1974; LeppelsacK and VogT, 1976), no such experiments were done in this study. An attempt to search for complex units has been made among the cortical units which did not respond to any standard sets of stimuli separately. Responses in these complex cortical units were studied only by means of artificial stimuli, i.e., combinations of pure tone bursts, white noise bursts, and FM tones. Increased response to pure tone with a background of continuous white noise may be due to a raising of basic activity by the white noise. It is likely that so-called complex units do not necessarily represent a higher order of information processing.

Vol. 30, No. 3, 1980 
Each response of several neurons in the present study was obviously unstable during the recording session. The discharge pattern evoked by repetitive stimulus could change from moment to moment. This is one reason why histograms were used in the analysis. In some neurons, however, the lability was slight, and some others were quite stable, such as the unit of Fig. 5.

Thanks go to Profs. Y. Katsuki and N. Saito for their support and Dr. R. C. Goris for correcting the manuscript.

\section{REFERENCES}

Abeles, M. and Goldstein, M. H., Jr. (1970) Functional architecture in cat primary auditory cortex: Columnar organization and organization according to depth. J. Neurophysiol., 33: 172-187.

Evans, E. F. and Whitfield, I. C. (1964) Classification of unit responses in the auditory cortex of the unanesthetized and unrestrained cat. J. Physiol. (Lond.), 171: 476-493.

Funkenstein, H. H. and Winter, P. (1973) Responses to acoustic stimuli of units in the auditory cortex of awakened squirrel monkeys. Exp. Brain Res., 18: 464-488.

Goldstein, M. H., Jr., Hall, J. L., II, and Butterfield, B. O. (1968) Single-unit activity in the primary auditory cortex of unanesthetized cats. J. Acoust. Soc. Am., 43: 444-455.

Goldstein, M. H., Jr., Abeles, M., Daly, R. L., and McIntosh, J. (1970) Functional architecture in cat primary auditory cortex. J. Neurophysiol., 33: 188-197.

Goldstern, M. H., Jr., and Abeles, M. (1975) Single unit activity of the auditory cortex. In Handbook of Sensory Physiology, Vol. 5, Part 2, ed. by KeIDEL, W. D. and NefF, W. D. Springer-Verlag, Berlin, pp. 199-218.

Hubel, D. H. and Wiesel, T. N. (1965) Receptive field and functional architecture in two nonstriate visual area (18 and 19) of the cat. J. Neurophysiol., 28: 229-289.

Katsuki, Y., Watanabe, T., and Maruyama, N. (1959) Activity of auditory neuron in upper levels of brain of cat. J. Neurophysiol., 22: 343-359.

Katsuki, Y., SugA, N., and KanNo, Y. (1962) Neural mechanism of the peripheral and central auditory system in monkeys. J. Acoust. Soc. Am., 34: 1396-1410.

LEPPELSACK, H. J. and VoGT, M. (1976) Responses of auditory neurons in the forebrain of a songbird to stimulation with species-specific sounds. J. Comp. Physiol., 107: 263-274.

Oonishi, S. and KatsuKI, Y. (1965) Functional organization and integrative mechanism on the auditory cortex of the cat. Jpn. J. Physiol., 15: 342-365.

Sovijarvi, A. R. A. and Hyvarinen, J. (1974) Auditory cortical neurons in the cat sensitive to the direction of sound source movement. Brain Res., 73: 455-471.

SugA, N. (1965a) Responses of Cortical auditory neurones to frequency modulated sound in echo-locating bats. Nature, 206: 890-891.

Suga, N. (1965b) Functional properties of auditory neurones in the cortex of echo-locating bats. J. Physiol. (Lond.), 181: 671-700.

SugA, N. (1968) Analysis of frequency-modulated and complex sounds by single auditory neurones in bats. J. Physiol. (Lond.), 198: 51-80.

Watanabe, T. (1972) Fundamental study of the neural mechanism in cats subserving the feature extraction process of complex sounds. Jpn. J. Physiol., 22: 569-583.

Whitfield, I. C. and Evans, E. F. (1965) Responses of auditory cortical neurons to stimuli of changing frequency. J. Neurophysiol., 28: 655-672.

Winter, P. and FunkensteIn, H. H. (1973) The effect of species-specific vocalization on the discharge of auditory cortical cell in awake squirrel monkey. Exp. Brain Res., 18: 489-504.

WollberG, Z. and Newman, J. D. (1972) Auditory cortex of squirrel monkey: Response patterns of single cells to species-specific vocalizations. Science, 175: 212-214. 